\title{
THE VIDEOTAPE RENTAL MODEL
}

\author{
BARRY A. PASTERNACK AND ZVI DREZNER \\ California State University-Fullerton
}

\begin{abstract}
In this paper we study the situation faced by a videotape rental store which must decide how many copies of a new videotape release it should purchase for rental to customers. A model representing this process is developed and contrasted with the classical newsboy problem.
\end{abstract}

Keywords: Newsboy Problem, Video rentals.

\section{Introduction}

The tremendous growth in sales of video cassette recorders over the past 15 years has fueled a second industry: videotape rental stores. These establishments purchase prerecorded video tapes at prices generally ranging from $\$ 20$ to $\$ 80$, and rent these tapes to customers at fees typically ranging from $\$ 1$ to $\$ 3$ per day.

While the tapes rented by these stores include specialized tapes such as exercise, travel and cooking; the vast majority of the rental traffic involves cinematic art. Typically, videotapes of movies are sold to the public several months following release of the movie for theatrical distribution. Customer demand is naturally greatest at the time the tape is first released and gradually decreases thereafter. This decrease in demand is attributable to a number of factors, including: demand satisfied through rentals, competition from other titles, and demand satisfaction through other media such as cable and television showing of the release (typically a movie is made available for cable viewing a few months following the videotape release).

Given the fact that rental demand substantially decreases over time, the two problems faced by the operator of a videotape rental store are determining how many copies of a given release should be purchased and determining when these copies should be sold for salvage. As with many inventory problems, the videotape rental store operator must strike a balance between having too many tapes in inventory (and thereby incurring costs due to excess stock) with having too few tapes in inventory (and thereby lost rentals and goodwill costs). For example, an article in the March 25, 1998 edition of the Wall Street Journal written by Eben Shapiro, "Movies, Blockbuster Seeks a New deal with Hollywood," cites research done by Warner Brothers which indicated $20 \%$ of surveyed customers were unable to rent the movie they wanted. This corroborated earlier research done by Steve Roberts, a consultant to the videotape industry. In an Associated Press story by Anthony Marquez ("Disposable videotapes to be tested", Orange County Register, page A3, March 30, 1989), Roberts estimated that "retailers lose anywhere from 
5 percent to 20 percent in business because frustrated customers cannot find their first choice movie and leave without renting anything." That article went on to also quote John Power, president of the 2500 member American Video Association as stating "Our consumer surveys show that customers are generally happy and that $60-70 \%$ of the time they get their tape."

This problem is similar in certain respects to that faced by a car rental company with the principal difference being that demand for car rentals does not appear to follow the same decay pattern as videotape rentals and the car rental problem is often times complicated by the fact that a certain proportion of customers return the car to a location different from the place where rented. Due to the relatively short demand life for the tape, the problem also appears to posses some of the attributes of the single period inventory (newsboy) problem. While there has been much research done on this model, see, for example, Goodman and Moody (1970), Atkinson (1979), Eppen (1979), Pasternack (1980), Parlar and Goyal (1984), Pasternack (1985), and Pasternack and Drezner (1991), the videotape problem appears, at least on the surface, somewhat different due to the fact that the tapes are not consumed during the rental process.

One major advantage the videotape rental problem has over other stochastic inventory problems is the prior information available to the store operator. Theatrical reference data is readily available and a number of services exist for forecasting initial rental demand based on factors such as theatrical receipts, movie genre, and individual store demographics. In a similar vein, data on how tape demand decays over time is kept by sophisticated store operators for determining accurate depreciation schedules. For example, the March 25, 1998 Wall Street Journal article cites Rentrak Corporation, a Portland, Oregon video distributor that has a propriety information system that records each and every rental at its clients' stores.

The focus of this paper is to derive a methodology by which this data can be incorporated into determining initial and continuing optimal stocking levels for videotapes. A number of models are presented with the difference being in the assumptions regarding the demand distributions and salvage values. In the next section we consider the case of a generalized demand pattern. A model is developed in which all units purchased are sold for salvage $N$ periods after acquisition. The general problem can then be decomposed into the various time horizons between salvage opportunities with the model used to find the optimal stocking level for each horizon. We show that for this model the optimal stocking level formula is, in a sense, equivalent to the result for the newsboy problem. The difficulty in applying the results, however, rests in the computational complexity of the resulting demand distribution formula. Analytical results are obtained for the case of nonstochastic, exponentially decaying demand.

In Section 3 the case of a stationary demand distribution is examined. A formula for the optimal stocking level is derived and, for the case of constant salvage value, it is shown that one should either not stock the tape or hold the tapes stocked forever. 


\section{The Generalized Model}

Let

$f_{i}(x)=$ the probability density function of demand at period $i$

$p=$ the rental revenue per period for the tape

$g=$ goodwill cost per rental due to stockout (unavailability of the tape during a period)

$h=$ holding cost per tape per period

$c=$ purchase cost per tape

$s(i)=$ salvage value per tape at period $i(s(i)<c$ for all $i)$

$e^{\alpha}=$ discount rate per period $(\alpha<0)$.

An approach to modeling the rental process would be to assume that all tapes initially purchased are held until some time, $N$, at which point they are disposed of for salvage value. This approach, presents no loss in generality because if there are multiple points in time during which salvage of the tapes is possible, one can simply assume complete liquidation of inventory followed by starting the process over with a partial inventory purchased at the salvage value.

For this model let $T(Q, N)$ be the total expected profit if there are $Q$ units in inventory and they are held until period $N$ (at which point they are all sold for salvage.) Hence, we have

$$
\begin{aligned}
& T(Q, N)=\sum_{i=0}^{N} e^{\alpha i} \int_{0}^{Q} p x f_{i}(x) d x+ \\
& \sum_{i=0}^{N} e^{\alpha i} \int_{Q}^{\infty}[p Q-g(x-Q)] f_{i}(x) d x-c Q+e^{\alpha N} s(N) Q-\sum_{i=0}^{N} e^{\alpha i} h Q
\end{aligned}
$$

Letting $f^{N}(x)=\sum_{i=0}^{N} e^{\alpha i} f_{i}(x)$ yields:

$$
\begin{aligned}
& T(Q, N)=\int_{0}^{Q} p x f^{N}(x) d x+ \\
& \int_{Q}^{\infty}[p Q-g(x-Q)] f^{N}(x) d x-c Q+e^{\alpha N} s(N) Q-\sum_{i=0}^{N} e^{\alpha i} h Q
\end{aligned}
$$

Differentiating $T(Q, N)$ in equation (2) with respect to $Q$, and setting this partial derivative equal to 0 gives: 


$$
\begin{aligned}
& p Q f^{N}(Q)+(p+g) \int_{Q}^{\infty} f^{N}(x) d x-[p Q-g(Q-Q)] f^{N}(Q)- \\
& c+e^{\alpha N} s(N)-h \sum_{i=0}^{N} e^{\alpha i}=0
\end{aligned}
$$

Canceling terms in equation (3) yields:

$$
\int_{Q^{*}}^{\infty} f^{N}(x) d x=\frac{c-s(N) e^{\alpha N}+h \sum_{i=0}^{N} e^{\alpha i}}{p+g}
$$

Equation (4) is analogous to the optimal solution for the newsboy problem. The principal difference lies in the necessity to calculate a compound probability function $f^{N}(x)$. Unfortunately, for most probability functions a closed form solution for $f^{N}(x)$ is difficult to obtain.

\section{Exponential Decay in Demand}

One situation where closed form approximate results are obtainable is the case of exponential decay in average demand with the demand at each period being exactly equal to the average demand for that period. In order to discretisize the demand we use the Dirac Delta function which is defined as a limit of spike functions for which the integral under the curve is equal to 1 . This means using the density function:

$$
f_{i}(x)=\left\{\begin{array}{c|c}
\infty & x=K e^{\beta i} \\
0 & \text { otherwise }
\end{array}\right.
$$

with $K>0$ and $\beta<0$.

This Dirac delta function has the following property for any function $G(X)$ :

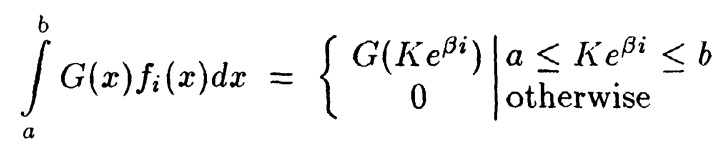

Note that since $\beta<0$ the mean of $f_{i}(x)$ decreases as $i$ increases. For this situation, the total profit is:

$$
\begin{aligned}
& T(Q, N)=\sum_{i=t+1}^{N} p K e^{\beta i} e^{\alpha i}+\sum_{i=0}^{t}\left[p Q-g\left(K e^{\beta i}-Q\right)\right] e^{\alpha i} \\
& -c Q+e^{\alpha N} s(N) Q-\sum_{i=0}^{N} e^{\alpha i} h Q
\end{aligned}
$$


where $t$ is defined as the largest integer value of $i$ for which $K e^{\beta i} \geq Q$.

Consider the left hand side integral of Equation (4):

$$
\int_{Q^{*}}^{\infty} f^{N}(x) d x=\sum_{i=0}^{N} e^{\alpha i} \int_{Q^{\cdot}}^{\infty} f_{i}(x)
$$

By applying Equation (5) to Equation (4) we observe that when $K e^{\beta i}<Q^{*}$ the integral of $f_{i}(x)$ is zero and therefore only terms for which $K e^{\beta i} \geq Q^{*}$ do not vanish in the sum. This yields a sum from $i=0$ to $i=t$. Now, when $K e^{\beta i} \geq Q^{*}$, the integral of $f_{i}(x)$ is 1 by Equation (5). This leads to:

$$
\sum_{0}^{t} e^{\alpha i}=\frac{c-s(N) e^{\alpha N}+h \sum_{i=0}^{N} e^{\alpha i}}{p+g}
$$

By summation of Equation (7):

$$
\frac{1-e^{\alpha(t+1)}}{1-e^{\alpha}}=\frac{c-s(N) e^{\alpha N}+h \sum_{i=0}^{N} e^{\alpha i}}{p+g}
$$

which leads to:

$$
e^{\alpha(t+1)}=1-\left(1-e^{\alpha}\right) \frac{c-s(N) e^{\alpha N}+h \sum_{i=0}^{N} e^{\alpha i}}{p+g}
$$

Define

$$
A=1-\left(1-e^{\alpha}\right) \frac{c-s(N) e^{\alpha N}+h \sum_{i=0}^{N} e^{\alpha i}}{p+g}
$$

Since $K e^{\beta t} \approx Q^{*}$, then $e^{\alpha t} \approx\left(\frac{Q^{*}}{K}\right)^{\frac{\alpha}{\beta}}$ which yields:

$$
Q^{*} \approx K\left\{e^{-\alpha} A\right\}^{\frac{\beta}{\alpha}}=K e^{-\beta} A^{\frac{\beta}{\alpha}}
$$

Equation (10) enables one to determine the approximately optimal $Q^{*}$ for a specific value of $N$. The best value of $N$ can be found by a simple search on the value of $N$ by substituting equation (10) into the total cost given by equation (6). 


\section{Stationary Demand}

In the case when demand is stationary, i.e. $f_{i}(x)=f(x)$ for all $i$, then equation (1) can be written as

$$
\begin{aligned}
& T(Q, N)=\frac{1-e^{\alpha(N+1)}}{1-e^{\alpha}}\left\{\int_{0}^{Q} p x f(x) d x+\int_{Q}^{\infty}[p Q-g(x-Q)] f(x) d x-h Q\right\} \\
& -c Q+e^{\alpha N} s(N) Q
\end{aligned}
$$

Differentiating (11) with respect to $Q$ and setting equal to 0 gives:

$$
F\left(Q^{*}\right)=\frac{p+g-h+\frac{1-e^{\alpha}}{1-e^{\alpha(N+1)}}\left(e^{\alpha N} s(N)-c\right)}{p+g}
$$

where the cumulative distribution function for the demand distribution is

$$
F(Q)=\int_{0}^{Q} f(x) d x
$$

In the case where $s(N)$ is constant, i.e. $s(N)=s$ for all $N$, then by equation (11) $T(Q, N)$ is of the form $A_{1}+A_{2} e^{\alpha N}$. Therefore, for a given $Q, T(Q, N)$ is either decreasing with $N$ or increasing with $N$ for all $N$. The solution is therefore either $N=\infty$, or $N=0$. This is stated as the following property.

THEOREM 1 For a given $Q$ and constant $s(N)$, The solution is either $N=0$ or $N=\infty$

Note that $N=0$ means to salvage all the tapes that have been bought immediately. In this case the value of $Q$ is irrelevant. The practical implication of this theorem is that if demand is stationary and salvage value is constant one should either not stock the tape or hold the tapes stocked in inventory forever. In this latter case, $N=\infty$. Hence, one can rewrite equation (12) as

$$
F\left(Q^{*}\right)=1-\frac{h+\left(1-e^{\alpha}\right) c}{p+g}
$$

and $Q^{*}$ can be obtained by solving it. Substitute $Q^{*}$ into equation (11) (for $N=\infty$ ) and if $T(Q, N)$ is negative, then $Q=0$ is optimal.

\section{Conclusion}

One of the most difficult aspects of any inventory problem is in forecasting the demand distribution. In the case of the videotape rental problem this task is eased 
somewhat by having knowledge regarding a movie's success in theatrical distribution. While the correlation between videotape and box office demand is not perfect, we believe that a reasonably accurate prediction of videotape rental demand can be gleaned from the experience of the movie in theatrical distribution. In a similar vein, the decay in average demand for the tape over time should not be too difficult to predict. We expect that an exponential decay function, such as that presented in Section 2, should give a reasonably accurate estimate of average demand during the first few months following the tape's initial release, with a constant demand pattern holding true thereafter. In light of this and the current inventory salvage practices we recommend the following three phase procedure for solving this inventory problem:

\section{Phase 1}

Determine the optimal stocking level during the first 30 days using a salvage value equal to approximately $40 \%$ of the tapes' initial cost. For this period estimate $K_{1}$ and $\beta_{1}$ for the mean decay function. It is difficult to come up with analytical solutions to equation (4) (the case of generalized demand) and numerical techniques are required. Therefore, we suggest modeling the process assuming deterministically decaying demand and finding the inventory level, $Q^{*}$, through equation (10).

\section{Phase 2}

For the next $T_{2}$ periods assume the mean period demand decays at an exponential rate with parameters $K=K_{1} e^{30 \beta_{1}}$ and $\beta=\beta_{2}$. Let the tape's cost equal the salvage value used in Phase 1 . The salvage value for the tape will be some constant, $s_{2}$. Again, assuming deterministically decaying demand one can estimate the length of this second phase and find the approximately optimal inventory level $Q^{*}$, through equation (10).

\section{Phase 3}

For the remaining time assume that the mean demand is constant at $\mu \approx K_{1} e^{30 \beta_{1}+T_{2} \beta_{2}}$ (where $T_{2}$ is the length of Phase 2). Both the initial cost of the tapes and salvage value are equal to $s_{2}$, the salvage value used in Phase 2. Using Property 1 and the discussion thereafter one can determine if it pays to keep the tape in inventory and, if it does, one can use equation (13) to determine the stocking level.

While this three phased technique will not be guaranteed to give the optimal stocking level for videotapes we believe that it offers a significant improvement over the nonanalytical methods currently in use. 


\section{References}

1. Atkinson, A.A. (1979), "Incentives, Uncertainty and Risk in the Newsboy Problem," Decision Sciences, 10, 341-357.

2. Eppen, G.D. (1979), "Effects of Centralization on Expected Costs in a Multilocation Newsboy Problem," Management Science, 25, 498-501.

3. Goodman, D.A. and K.W. Moody (1970), "Determining Optimum Price Promotion Quantities, Journal of Marketing, 34, 31-39.

4. Parlar, M and Goyal, S.K. (1984), "Optimal Ordering Decisions for Two Substitutable Products with Stochastic Demands," OPSEARCH, 21, 1-15.

5. Pasternack, Barry A. (1980), "Filling Out the Doughnut: The Single Period Inventory Model in Corporate Pricing Policy," Interfaces, 10, 96-100.

6. Pasternack, Barry A. (1985), "Optimal Pricing and Return Policies for Perishable Commodities," Marketing Science, 4, 166-176.

7. Pasternack, B. A and Drezner, Z. (1991), "Optimal Inventory Policies for Substitutable Commodities", Naval Research Logistics, 38, 221-240. 


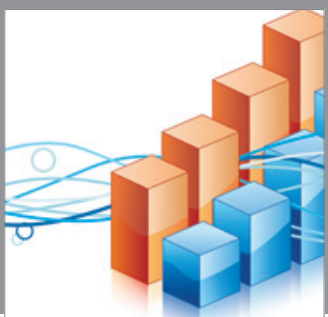

Advances in

Operations Research

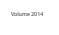

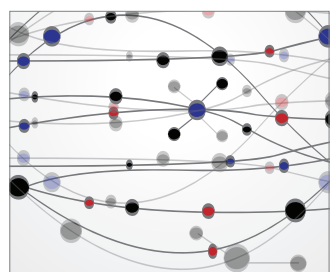

\section{The Scientific} World Journal
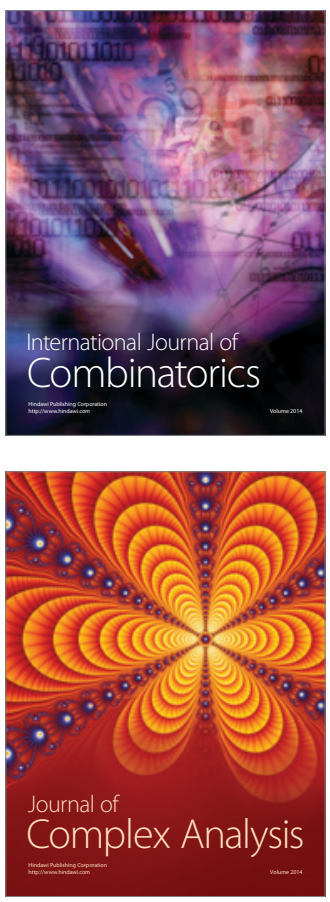

International Journal of

Mathematics and

Mathematical

Sciences
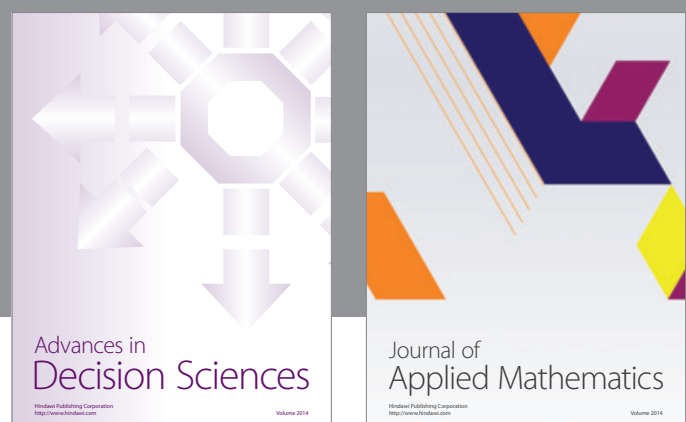

Journal of

Applied Mathematics
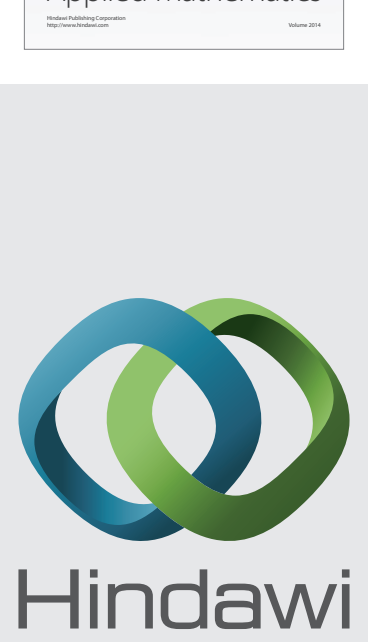

Submit your manuscripts at http://www.hindawi.com
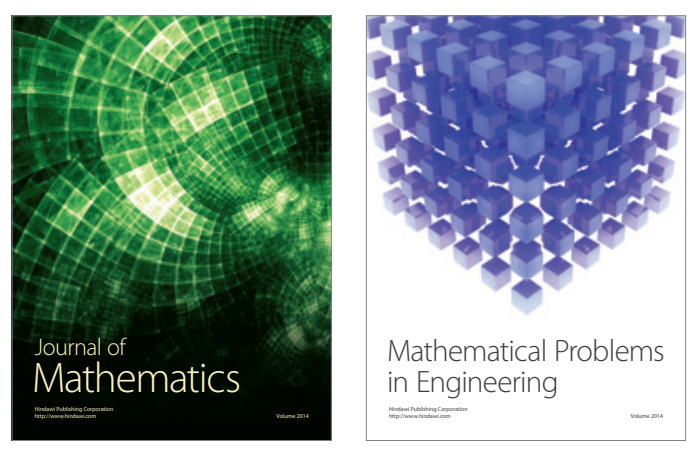

Mathematical Problems in Engineering
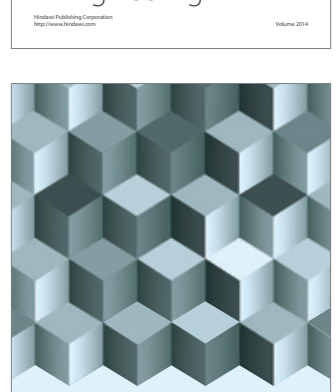

Journal of

Function Spaces
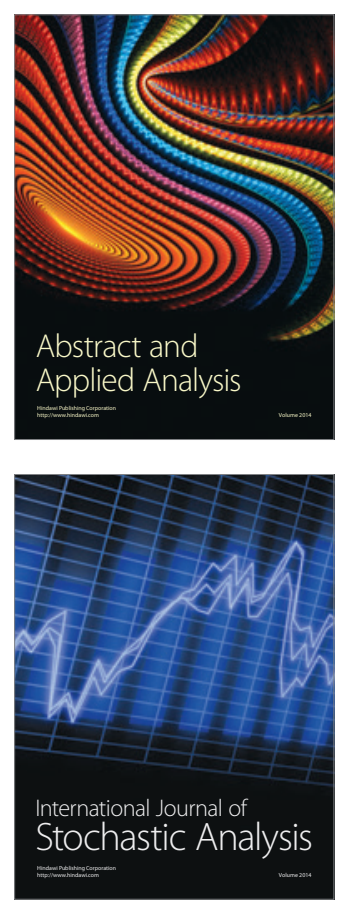

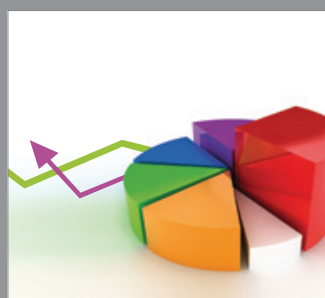

ournal of

Probability and Statistics

Promensencen
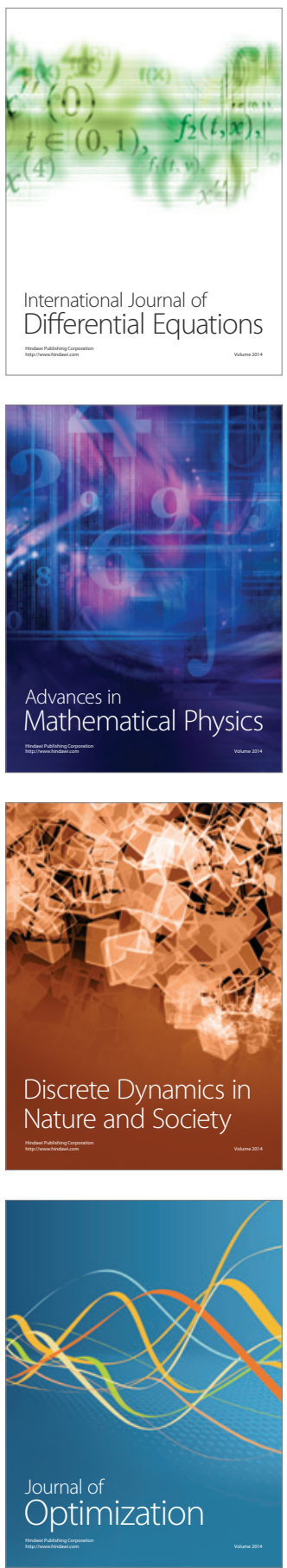